\title{
Reticular reflex myoclonus: a physiological type of human post-hypoxic myoclonus
}

\author{
M. HALLETT, ${ }^{1}$ D. CHADWICK, JANE ADAM, AND C. D. MARSDEN ${ }^{2}$ \\ From the University Department of Neurology, King's College Hospital Medical School, \\ and Institute of Psychiatry, London
}

SUMMARY A patient with post-hypoxic myoclonus, sensitive to therapy with 5-hydroxytryptophan and clonazepam, was subjected to detailed electrophysiological investigation. Brief generalised jerks followed the critical stimulus of muscle stretch. The electroencephalogram showed generalised spikes that were associated with, but not time locked to, the myoclonus. The cranial nerve nuclei were activated upward. Analysis of the findings suggests that the mechanism of the myoclonus is hyperactivity of a reflex mediated in the reticular formation of the medulla oblongata.

Myoclonus is a complicated and ill-understood phenomenon associated with a variety of electrophysiological abnormalities (Halliday, 1967) and caused by many pathological conditions (Marsden and Parkes, 1973). No one pathophysiological mechanism can account for all the types of myoclonus seen in clinical practice. In some patients the myoclonic jerks occur spontaneously while the subject is at rest. In others they appear only on action and in such patients myoclonic jerks may be triggered by sensory stimuli. Such reflex myoclonus is seen in response to visual or auditory stimuli. Occasionally a tendon tap will elicit a myoclonic response, in which case the critical stimulus may be muscle stretch, a joint movement, or cutaneous stimulation. In one particularly well-studied case under the care of Dr A. Carmichael at the National Hospital, Queen Square, careful clinical observation by Dr Alan Norton established the critical stimulus to be muscle stretch. This patient was found to have giant cortical potentials evoked by electrical stimulation of peripheral nerves (Dawson, 1947), stimuli which also caused myoclonic jerks.

Recently, Sutton and Mayer (1974) have described a patient with focal reflex myoclonus due to cerebral vascular disease with infarction in the left cerebral hemisphere. She suffered from intermittent jerking

\footnotetext{
${ }^{1}$ Moseley Travelling Fellow of Harvard Medical School.

Present address: Section of Neurology, Peter Bent Brigham Hospital, Boston, Mass 02115, USA.

${ }^{2}$ Address for correspondence: C. D. Marsden, Institute of Psychiatry, De Crespigny Park, Denmark Hill, London SE5.

Accepted 23 August 1976
}

of the right fingers and hand, and to a lesser extent the right face, jaw, tongue, and foot. The onset was acute at the age of 66 years and was associated initially with a mild right hemiparesis and hemisensory loss, as well as speech disturbance and hemianopia. When studied seven years after the onset, it was noted that the myoclonic jerks of the right limbs increased on voluntary action and were precipitated by eliciting tendon jerks. Electrophysiological investigation revealed that stimulation of the median nerve at the wrist or the posterior tibial nerve at the ankle evoked a late myoclonic jerk (termed $\mathrm{C}$ reflex in the original paper). The latency recorded from the thenar muscles from stimulating the median nerve at the wrist was some $51 \mathrm{~ms}$. When the response was recorded in plantar muscles after stimulating the posterior tibial nerve at the ankle, the latency was some $103 \mathrm{~ms}$. The stimulus at either site evoked abnormal large somatosensory cortical potentials recorded from the opposite hemisphere. The authors concluded that this reflexly evoked myoclonic jerk occurred after an interval sufficient to allow passage of afferent impulses to the cerebral cortex and efferent impulses from cortex down the corticospinal tract to motoneurones. In a subsequent article, Sutton (1975) analysed the critical stimulus required to elicit reflex myoclonus in this patient. Touch or pressure, in the absence of movement, was an adequate stimulus, whereas muscle stretch by itself was not, in keeping with the observation that reflex myoclonus could be induced as easily by stimulation of the digital nerves as by stimulation of the median nerve at the wrist.

The case described by Carmichael and Dawson is 
similar to that described by Sutton and Mayer in that both showed reflex myoclonus in response to a tendon tap or to mixed nerve stimulation, and both had giant cortical potentials evoked by the latter stimulus. The two cases differ in that the critical stimulus in the first case was stretch of muscle while in the latter it was touch or pressure.

In the present study, we have investigated a patient with post-anoxic reflex myoclonus in whom the critical stimulus would appear to be stretch or movement, rather than touch or pressure, and in whom cortical evoked potentials were not abnormally large.

\section{Case history}

A 55 year old man, resident in Bermuda for 20 years, but originating from Great Britain, had been asthmatic for 15 years. He had sustained a period of respiratory and cardiac arrest during an attack of asthma six months previously. After this he was unconscious for two weeks before his level of consciousness gradually improved. However, as he improved, it was noticed that all four limbs were rigid and akinetic, and that there were continuous involuntary movements affecting all four limbs, head, and trunk. His involuntary movements had persisted despite treatment with phenobarbitone and diphenylhydantoin and during the six months between the initial period of anoxia and investigation he had had two generalised convulsions.

On examination he was alert but disorientated in time and space. Spontaneous speech was simple in form and he showed a moderate mixed dysphasia with perseveration, as well as a marked slurring dysarthria. He was markedly bradykinetic and muscle tone was increased in all limbs, having the characteristics both of extrapyramidal rigidity and spasticity. His facial expression was rather fixed, and vertical gaze restricted. His overall posture was one of flexion, particularly of the trunk, neck, elbows, and hips, and there were marked bilateral grasp and rooting reflexes. His tendon jerks were brisk throughout and plantar responses extensor. Myoclonic jerks occurred spontaneously and in response to stimuli, and will be described in detail below.

Investigation revealed a normal haemoglobin and white blood cell count, with an ESR of $42 \mathrm{~mm} / \mathrm{h}$. Serum urea and electrolytes, calcium, inorganic phosphate, and liver function were all normal. Skull and chest radiographs and ECG were within normal limits. CSF obtained by routine lumbar puncture showed no cells and a normal protein content. CSF 5-hydroxyindolacetic acid was $23.7 \mathrm{ng} / \mathrm{ml}$ (control: $32.6 \pm 9.6 \mathrm{ng} / \mathrm{ml}($ mean $\pm \mathrm{SD}))$ and CSF homo- vanillic acid was $26.7 \mathrm{ng} / \mathrm{ml}$ (control: $52.3 \pm 30 . \frac{\underline{z}}{\mathrm{z}}$ $\mathrm{ng} / \mathrm{ml}$ ).

\section{PROCEDURE}

The electromyogram and electroencephalogran? were recorded from various sites using surfacen electrodes. Signals were preamplified by Devices 316 amplifiers and fed into a PDP 12 computer. Spon耐 taneous myoclonic jerks were recorded using the programme PASTIME triggering the computer from the EMG of one of the muscles in order to? observe events both before and after the trigger Elicited jerks were recorded using the programme $A$ triggering the computer at the time of delivery of the stimulus. Data were collected in single trials or in averages of up to 128 trials.

Averaged data gave inconsistent results on occasions; this turned out to be due to jitter in the timing of EMG bursts in different muscles with respect to each other, and to jitter of the bursts ing different muscles with respect to the stimulus. Thus most of the results presented were taken from seriesp of single trials.

The differences in the time of onset of the EM 0 is burst in one muscle with respect to another museles (or the stimulus) was taken as the average differercece in those trials where both muscles were active and time of onset could be determined clearly. The standard deviation of the individual differences wast taken as a measure of the jitter.

\section{Results}

\section{NERVE CONDUCTION STUDIES}

In order to derive motor and sensory conductions times, peripheral nerve conduction velocities, mono synaptic tendon jerks responses, distal latencies and the latencies of $F$ waves and $H$ reflexes were obtained in the standard manner.

The minimum time needed for a neural signal tog travel down a limb from one muscle to the next was calculated from motor nerve stimulation (Table 1) That these times were appropriate in certain physio logical circumstances was confirmed using the monosynaptic tendon jerk data. For example, the tendon jerk latency in biceps to a tap on the biceps tendon was $15 \mathrm{~ms}$ and in finger flexors to a finger tap was $21 \mathrm{~ms}$. The difference, $6 \mathrm{~ms}$, represented the afferent and efferent time from finger flexors to biceps. Assuming that motor and afferent nerve conduction velocities were approximately the same $3 \mathrm{~ms}$ was the one-way time from finger flexors to biceps, a figure confirming the result obtained with direct motor nerve stimulation. A similar calculation using the latencies of the quadriceps jerk $(25 \mathrm{~ms}$ R and soleus jerk ( $43 \mathrm{~ms})$ gave $9 \mathrm{~ms}$ for the time between 
Table 1 Latencies in ms from muscle to muscle based on (1) supramaximal motor nerve stimulation, (2) spontaneous jerks, (3) jerks elicited by either taps to the toes (evoking jerks in leg), taps to fingers (evoking jerks in arm) or median nerve stimulation at wrist

\begin{tabular}{|c|c|c|c|c|c|}
\hline & $\begin{array}{l}\text { Motor nerve } \\
\text { stimulation }\end{array}$ & $\begin{array}{l}\text { Spontaneous } \\
\text { jerks }\end{array}$ & Toe taps & Finger taps & $\begin{array}{l}\text { Median nerve } \\
\text { stimulation }\end{array}$ \\
\hline Biceps to finger flexors & 3 & $4 \pm 4(12)^{*}$ & - & 2 & $0 \pm 6(12)$ \\
\hline Finger flexors to abductor pollicis brevis & 6 & $10 \pm 4(12)$ & - & 二 & $9 \pm 3(13)$ \\
\hline Biceps femoris to soleus & 6 & $10 \pm 3(27)$ & $14 \pm 6(16)$ & - & $10 \pm 6(7)$ \\
\hline Soleus to flexor hallucis brevis & 11 & $14 \pm 3(14)$ & $7 \pm 8(14)$ & - & $13 \pm 4(8)$ \\
\hline 'Biceps to biceps femoris' & - & $5 \pm 6(14)$ & - & - & $8 \pm 13(8)$ \\
\hline
\end{tabular}

* Mean latency ( $\pm 1 \mathrm{SD}$ ) is given in ms and is based on a number of observations (in parentheses) of single records in which both muscles were active, except in the case of finger taps where only averaged records were available.

The mean difference between a pair of muscles shown in Table 1 may differ from that shown in Table 3, in which latencies from stimuli were measured in all records in which the particular muscle was active, irrespective of whether the other muscle fired. These minor differences reflect the jitter of latency for a given muscle with respect to the stimulus and to other muscles.

upper leg and calf, slightly longer than the fastest motor conduction time of $6 \mathrm{~ms}$.

\section{SPONTANEOUS JERKS}

\section{Clinical observations}

Myoclonic jerks were present at rest, were intensified by attempted voluntary and passive movement, were diminished during drowsiness or light sleep, and were absent in deep sleep. The jerks occurred irregularly, on average five to 10 times per minute and usually involved the whole body, although occasionally the jerk was limited to a single limb or part of a limb. Muscles of the four limbs, head, neck, trunk, and face were involved in the myoclonus. Depending on the amount of activity in particular muscles, the jerks would vary in clinical form, but flexors tended to be more active than extensors. Commonly there would be nodding of the head, bending of the trunk, flexion of the arms, shrugging of the shoulders, and flexion withdrawal of the legs.

\section{EMG studies}

Electrophysiological surveys were made of the cranial nerve musculature and the four limbs. Large, relatively synchronous bursts of activity in the muscles corresponded to the clinical jerks (Fig.1). In most spontaneous jerks all muscles surveyed participated regardless of the clinical pattern.

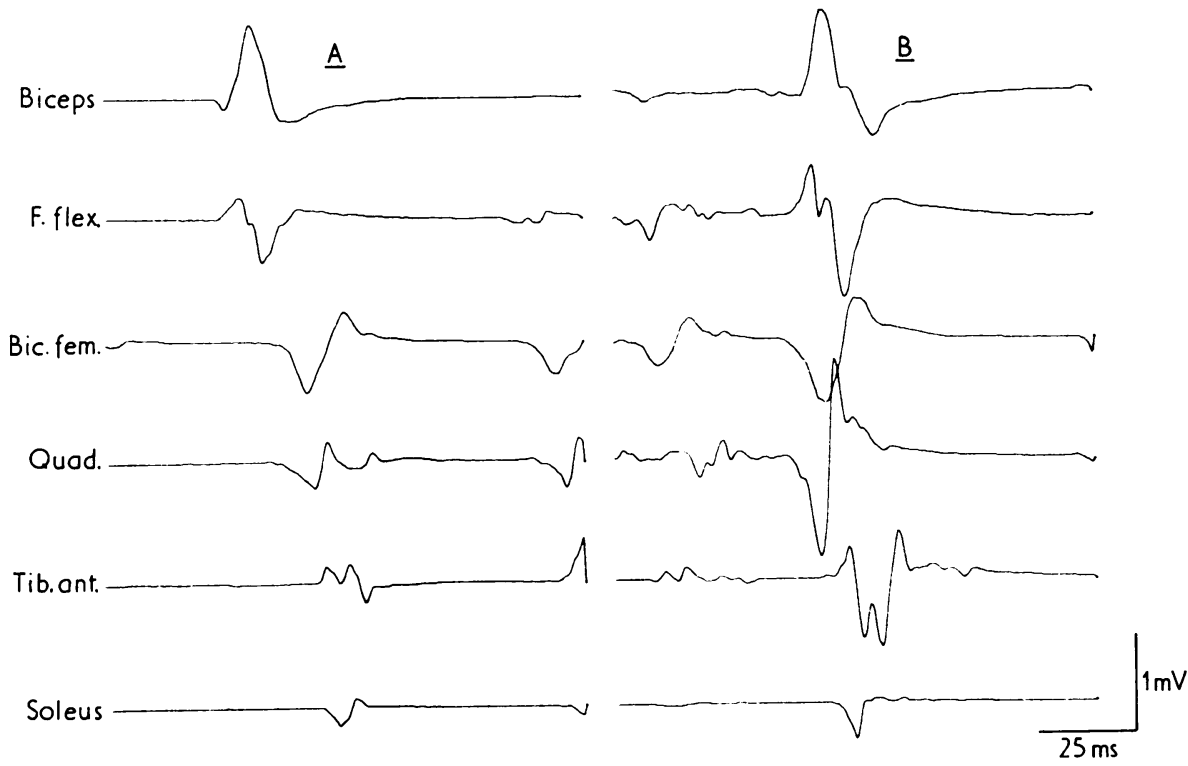

Fig. 1 Electromyographic recording from right limbs of two spontaneous myoclonic jerks. Muscles recorded from are biceps, finger flexors ( $F$. flex.), biceps femoris (Bic. fem.) quadriceps (Quad.), tibialis anterior (Tib. ant.), and soleus. In part $A$, the jerk in the arm precedes that in the leg, while, in part $B$, the earliest activity in leg precedes that in the arm. 
Usually both components of an agonist-antagonist pair were active, but, corresponding to the clinical impression of flexor predominance, the amplitude of the flexor component was usually greater than the amplitude of the extensor and at times the extensor component was apparently absent. The EMG of a single jerk was simple in form, having only a few phases and lasting $10-30 \mathrm{~ms}$. The time of onset of activity in one muscle with respect to another of a flexor-extensor pair varied by about $3 \mathrm{~ms}$. The latency from proximal to distal muscles in one limb in individual records varied by $4-5 \mathrm{~ms}$. Despite this jitter, the average time of one muscle's onset with response to another was approximately proportional to the distance of the muscles from the neuraxis. By using the average difference in time of the onset of the EMG in different muscles during the spontaneous jerks, it was possible to calculate 'motor conduction times' from one muscle to the next; these times were somewhat slower than the maximal motor conduction times obtained by direct nerve stimulation (Table 1).

The same muscles in different limbs had a 6-8 ms jitter with respect to each other; this applied to the two arms, the two legs, or one arm and the ipsilateral leg. On average, one side of the body did not become active earlier than the other. The arms tended to precede the legs, but only by 5-6 ms (biceps with respect to biceps femoris), and in some jerks the legs preceded the arms. The cranial nerve musculature, at least that supplied by the lower cranial nerves, tended to precede the limbs (Fig. 2 and Table 2). The cranial nerve nuclei seemed to be activated in ascending order.

\section{EEG studies}

The EEG showed predominant small amplitude intermediate slow and fast activity over both hemispheres without any definite alpha rhythm. There were very frequent spikes, often doublets or triplets, followed by slow waves. The spikes were usually triphasic, with successive positive, negative and positive waves (Figs. 2-5); they were generalised in distribution with slightly higher amplitude at the vertex (Fig. 3). The spikes were usually, but not always, associated with the myoclonic jerks; there was a marked variation in the timing of the spike (measured at the beginning of the initial positive component) and the onset of the EMG activity. Activity of the lower cranial nerve musculature (sternocleidomastoid and trapezius), but not of the upper cranial nerve musculature (orbicularis oris and masseter), usually preceded the cortical spike (Table 2). Even the activity of the leg muscles preceded the cortical spikes at times. The failure of the EEG spikes to be tightly time-locked to the EMG discharges was illustrated by the effect of

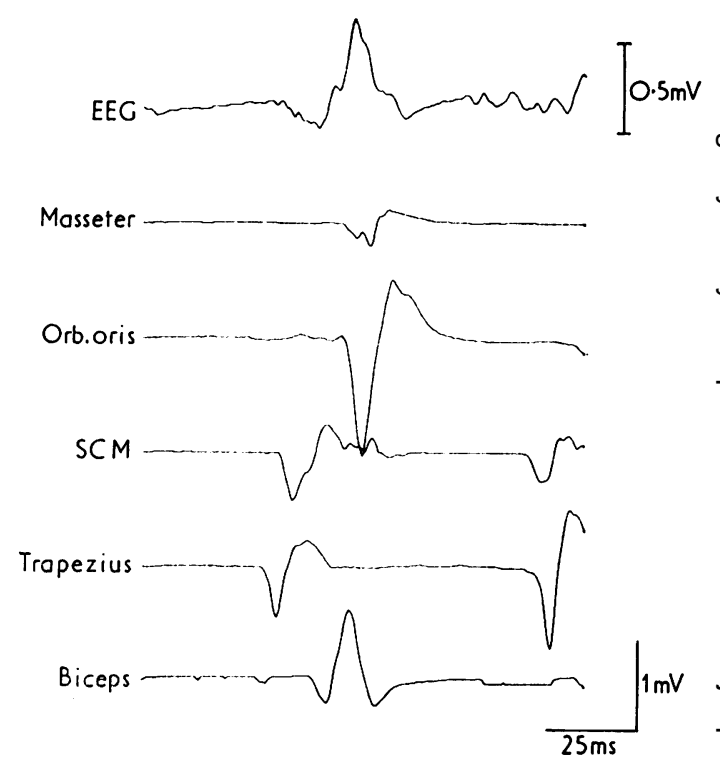

Fig. 2 Electrical record of spontaneous myoclonic jerk including activity in cranial nerve muscles. The electroencephalographic recording (EEG) is from a point $1 \mathrm{~cm}$ to the left and $2 \mathrm{~cm}$ behind the vertex referred to a mid-frontal electrode (a positive deflection is downward). Other records are from the right masseter, left orbicular oris (Orb. oris), left sternocleidomastoid $(S C M)$, right trapezius, and right biceps. Note the activation of the cranial nerves up the brain stem, and the onset of activity in trapezius before that in the EEG. The upper voltage calibration refers to the EEG record and the lower voltage calibration to all of the EMG records.

Table 2 Spontaneous jerks in cranial nerve musculature

$\begin{array}{lr}\text { Sternocleidomastoid } & 3 \pm 3(26) \\ \text { Orbicularis oris } & 10 \pm 6(24) \\ \text { Masseter } & 19 \pm 4(16) \\ \text { EEG spike (initial positive) } & 4 \pm 5(25) \\ \text { Biceps } & 11 \pm 4(24)\end{array}$

The onset of activity in a given muscle or the time of onset of the initial positive wave in the EEG, was measured from the time of onset of activity in trapezius.

Mean latency in $\mathrm{ms}( \pm 1 \mathrm{SD})$ is shown for a number of observations (in parentheses).

averaging the EEG by triggering the averaging sweep from EMG bursts in an active muscle (Fig. 4). No EEG activity averaged with respect to the myoclonic muscle jerks.

Cortical evoked responses in this patient after median nerve stimulation at the wrist were not ab- N normally large. Only contralateral potentials could be identified; the initial positive phase was $4 \mu \mathrm{V}$ in $\mathcal{O}$ amplitude after left median nerve stimulation and $\mathrm{N}$ 


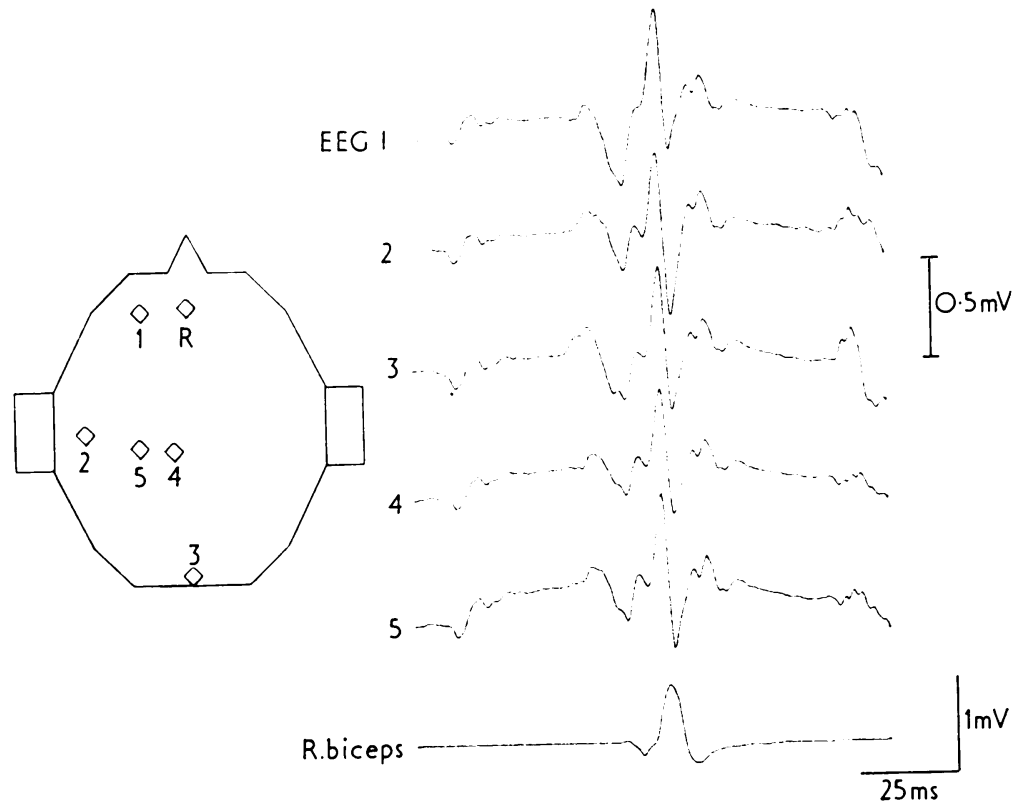

Fig. 3 Electroencephalographic correlate of a spontaneous myoclonic jerk. The electroencephalogram is sampled from five widely separated sites in the left hemisphere as indicated in the diagram and referred to a mid-frontal electrode $(R)(a$ positive deflection is downward). Activity in the right biceps is taken as a marker of the myoclonic jerk. It is apparent that the electrocortical spike activity is widespread. The upper voltage calibration marker refers to all of the EEG records and the lower voltage calibration marker refers to the EMG record.

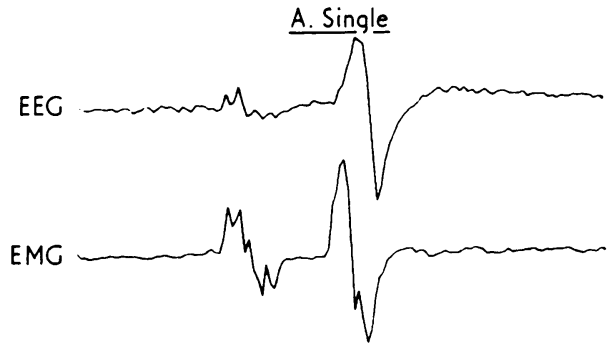

B. Average

EEC

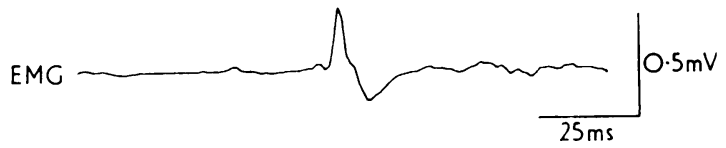

Fig. 4 The EEG-EMG correlation of the myoclonus. In each part of the diagram the EEG is recorded from the vertex referred to the left ear (a positive deflection is downward), and the EMG is from the left sternocleidomastoid muscle. Part A shows the events in a single spontaneous jerk. Part B is the average of 84 spontaneous jerks with the averaging being triggered on the occurrence of the EMG event. Since the EEG record in part $B$ shows essentially no activity, the timing of the EEG spike seen in part $A$ must vary considerably with respect to the EMG jerk.
$5 \mu \mathrm{V}$ after right median nerve stimulation. The latency to onset of these potentials was $29 \mathrm{~ms}$ and 22 ms respectively. The flash evoked visual response and the auditory evoked potential were similarly not exceptional in size or latency.

\section{ELICITED JERKS}

\section{Clinical observations}

Myoclonic jerks could be precipitated by a variety of sensory stimuli, including startle, an abrupt noise, or a tendon tap. In the latter case, light taps might evoke jerks confined to the stimulated limb, but usually involved the whole body. With regard to the sensitivity to a tendon tap, the arms were much more sensitive than the legs, and the hands and feet were much more sensitive than proximal structures. The most reactive site was the fingers. A light tap delivered to the tips of the fingers or thumb to elicit a finger jerk would regularly cause a myoclonic jerk. Taps to the tendons of biceps or triceps rarely caused jerks. Taps to the pads of the toes of ten caused jerks, but taps to the Achilles tendon and patellar tendon were ineffective. Careful analysis of the adequate stimulus required to elicit a myoclonic jerk in response to stimulation of the fingers or thumb revealed that pin prick, light touch, or deep touch to the tips of the fingers were ineffective. Even a brisk tap with a tendon hammer on the pads of the fingers did not necessarily cause jerks when they were firmly supported on a flat surface to prevent movement at joints. However, a tap to the 

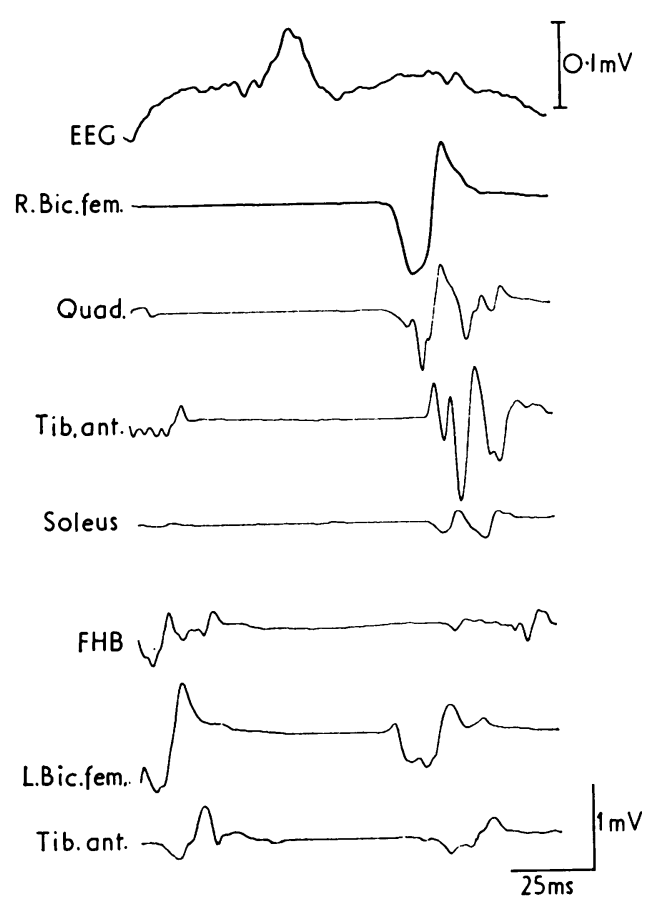

Fig. 5 Electrical events of a myoclonic jerk evoked by a sharp tap to the under surface of the right toes. The $E E G$ is recorded from an electrode $1 \mathrm{~cm}$ to the left and $2 \mathrm{~cm}$ behind the vertex and referred to a mid-frontal electrode (a positive deflection is downward). The muscles recorded from are right biceps femoris (R. Bic. fem.), right quadriceps (Quad.), right tibialis anterior (Tib. ant.), right soleus, right flexor hallucis brevis (FHB), left biceps femoris (L. Bic. fem.) and left tibialis anterior Tib. ant.). The upper voltage calibration marker refers to the EEG while the lower marker refers to all of the EMG records.

finger tips such as to elicit a tendon jerk regularly caused a myoclonic response.

\footnotetext{
Muscle stretch

A tendon hammer, incorporating a triggering device, was used in the usual clinical manner to stretch the finger flexors, biceps, triceps, and toe (and foot) plantar flexors. The resulting EMG responses correlated with the visible myoclonic jerks evoked and were similar in appearance to those seen with the spontaneous jerks.

Single responses to taps to the toes were studied extensively (Fig. 5). The EMG activity elicited with this stimulus was most prominent in the ipsilateral lower leg, but was usually seen in the upper leg as well. A larger amplitude of EMG activity in flexor muscles (similar to spontaneous jerks) correlated with the
}

clinical appearance of flexion at hip and knee and dorsiflexion at the ankle. Activity was frequently seen in the opposite leg and sometimes in the ipsilateral arm, but this latter response was so erratic that it was uncertain whether it was part of the myoclonic jerk being studied. The latency of response from the stimulus to the individual muscles was markedly variable with a jitter of 9 to $13 \mathrm{~ms}$ (Table 3). The jitter of a muscle to its antagonist was about $3 \mathrm{~ms}$, the jitter to another muscle in the same limb was about $5 \mathrm{~ms}$, and the jitter to the same muscle in the opposite limb about $9 \mathrm{~ms}$. All these values were similar to those obtained with the spontaneous jerks. Within the same limb the average time of onset of the EMG varied with the distance from the neuraxis; 'conduction times' from one muscle to another could be derived and were generally similar to those found for spontaneous jerks (Table 1). Only averaged data were available for the myoclonic jerks produced by taps to the fingers (Fig. 6a). Responses were seen only in the biceps and finger flexors of the limb being stimulated. Derived conduction time between these two muscles was similar to that obtained by other methods (Table 1) and latencies of response were similar to those ob-o $\dot{\omega}$ tained by mixed nerve stimulation (see below and Table 3).

\section{Mixed nerve stimulation}

Direct electrical stimulation of the median nerve a the wrist or elbow was a very effective stimulus for myoclonic jerks, whereas stimulation of the posterio tibial nerve at the ankle gave inconsistent responses As with spontaneous jerks and those produced by muscle stretch, the electromyographic correlate of the myoclonus was a large, relatively synchronous burst of activity in the muscles. The results of stimulation of the median nerve at the wrist were studied in detail (Fig. 7). In the figure, there are three responses in abductor pollicis brevis. The first is the direct muscle response ( $M$ response) to the nerve stimulation, which has overloaded the amplifier at the gain employed. This is followed by the $F$ wave. Last, corresponding in time with activity in the other muscles, is the myoclonic burst of activity. The myoclonus was widespread with a regular response in all the muscles of the same arm and usually in the ipsilateral leg. The latency of response (Table 3 ) in the various muscles of the arm showed a variation of 4-8 ms and in the leg of 11-15 ms. The jitter of various muscle pairs with respect to each other and the 'motor conduction times' found by considering onset of activity for muscles within the same limb (Table 1) were generally similar to those calculated from records of spontaneous jerks or those elicited by muscle stretch. The arms (biceps) preceded the legs (biceps femoris) by $8 \pm 13 \mathrm{~ms}$. For median nerve stimulation at the 

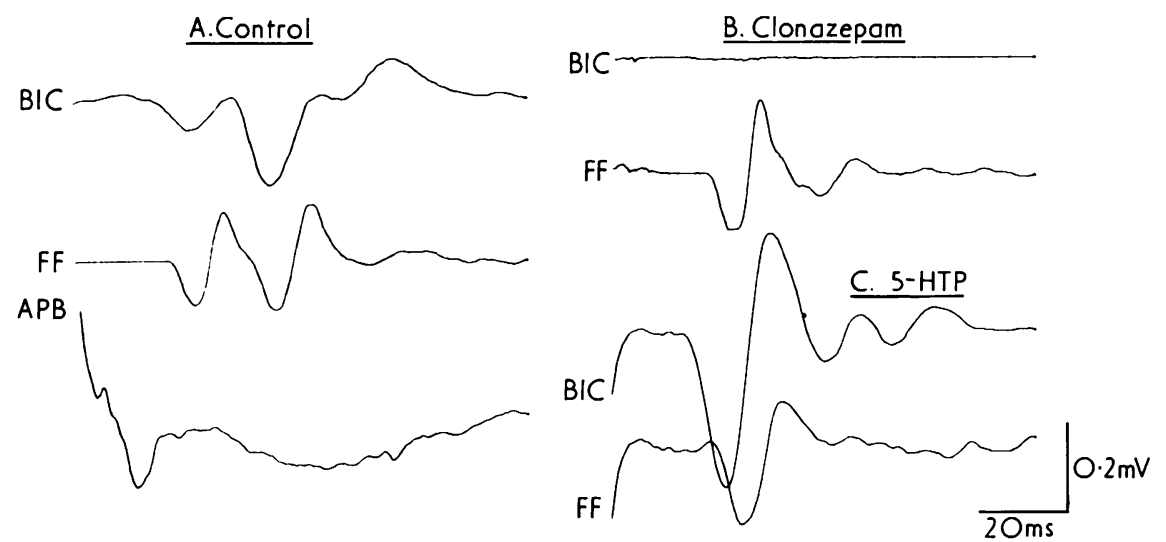

Fig. 6 Myoclonic responses to finger tap (sudden extension of the fingers). The records, which are averages of 64 trials, are taken from biceps (BIC), finger flexors (FF), and abductor pollicis brevis (APB). Part $A$ is the control, part B is after the administration of clonazepam, and part $C$ is after the administration of 5-HTP (see text for details). Because $A P B$ showed no averaged myoclonic activity even on the control run, it is not illustrated in parts $B$ and $C$. Note in the control record the initial burst of activity at tendon jerk latency in finger flexors, which is followed by the myoclonic burst. After clonazepam, the tendon jerk is unaffected, but the myoclonic burst is absent or reduced. After 5-HTP, the tendon jerk is enhanced, but the myoclonic burst is reduced.

Table 3 Latencies for elicited jerks

\begin{tabular}{llll}
\hline & Toe taps & Finger taps & $\begin{array}{l}\text { Median } \\
\text { nerve shocks }\end{array}$ \\
\hline Biceps & & 35 & $39 \pm 4(12)$ \\
Triceps & & No response & $43 \pm 8(16)$ \\
Finger flexors & & 37 & $39 \pm 5(19)$ \\
Finger extensors & & No response & $43 \pm 5(19)$ \\
Abductor pollicis brevis & & No response & $49 \pm 4(13)$ \\
Biceps femoris & $74 \pm 9(16)$ & & $44 \pm 13(12)$ \\
Quadriceps & $75 \pm 9(15)$ & & Not studied \\
Tibialis anterior & $87 \pm 12(17)$ & & $58 \pm 15(8)$ \\
Soleus & $89 \pm 13(17)$ & & $70 \pm 11(11)$ \\
Flexor hallucis brevis & $96 \pm 11(14)$ & & Not studied \\
\hline
\end{tabular}

Mean latency ( $\pm 1 \mathrm{SD}$ ) is given in ms and is based on a number of observations (in parentheses), except in the case of finger taps where only averaged records were available. Latencies were measured in ms from the stimulus trigger produced by the tap or shock to the initial EMG deflection in the response evoked in the muscles stated.

wrist the motor threshold for EMG response in abductor pollicis brevis was about $80-90 \mathrm{~V}$ with a $100 \mu \mathrm{S}$ pulse. Myoclonic activity was obtained with voltages of $75 \%$ of threshold (Fig. 8a).

Conduction velocity in the peripheral part of the afferent path responsible for the myoclonus can be deduced by comparing the latency of the EMG activity after a distal and a proximal stimulus. Using averages of 32 single trials, the latency of the myoclonic activity in biceps was compared after median nerve stimulation at the wrist and elbow. The average latency to the onset of biceps activity was $33 \mathrm{~ms}$ in $\mathbf{2 5}$ averages of 32 trials after median nerve stimulation at the wrist and was $29.5 \mathrm{~ms}$ in five averages of 32 trials after stimulation at the elbow. The distance between the sites of stimulation was $28 \mathrm{~cm}$; thus the afferent conduction velocity was about $80 \mathrm{~m} / \mathrm{s}$.

\section{Sensory nerve stimulation}

Using ring electrodes the sensory nerves to the fingers were stimulated. The thumb, index, and middle fingers were stimulated separately with $100 \mu$ s pulses of up to $90 \mathrm{~V}$, parameters which were effective when stimulating the median nerve at the wrist (Fig. 8B). (The patient's mental state made it impossible to determine a sensory threshold.) With this form of stimulation myoclonic jerks were not seen clinically or in EMG records of single trials or averages of 32 .

\section{DRUG EFFECTS \\ Clonazepam}

An intravenous bolus of $1 \mathrm{mg}$ clonazepam was given. Within one minute the spontaneous myoclonus had stopped. Myoclonic jerks could no longer be averaged by tendon taps to the fingers or median nerve stimulation at the wrist $(100 \mu \mathrm{s}, 90 \mathrm{~V})$, conditions which were previously effective (Fig. $6 \mathrm{~b}$ and $9 \mathrm{~b}$ ).

\section{5-Hydroxytryptophan (5-HTP)}

The patient was given an intravenous infusion of 150 mg 5-HTP in $500 \mathrm{ml}$ of normal saline over two hours. At the end of the infusion the myoclonus had disappeared and was still absent five hours later at the time of physiological testing. The patient was nauseated, depressed, and rather drowsy, but was easily 


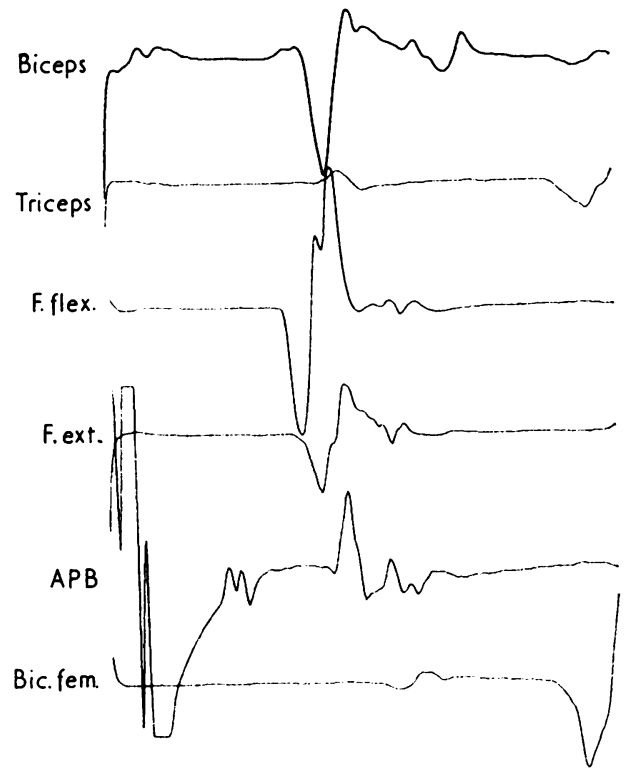

Tib.ont.

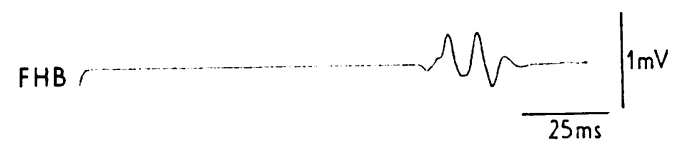

Fig. 7 Electromyographic recording of a myoclonic jerk in response to right median nerve stimulation at the wrist. Muscles recorded from, all on the right side, are biceps, triceps, finger flexors ( $F$. flex.), finger extensors (F. ext.), abductor pollicis brevis (APB), biceps femoris (Bic. fem.), tibialis anterior (Tib. ant.), and flexor hallucis brevis (FHB). In APB, three responses can be seen: the direct $M$ responses (which overload the amplifiers), an $F$ wave, and then the myoclonic jerk. aroused. Clinically the tendon jerks were enhanced. Averaged responses were obtained to tendon taps of the fingers (Fig. 6c) and to stimulation of the median nerve at the wrist (Fig. 9c) and were compared with the responses the previous day (with similar EMG electrode placement) before the drug was given. After the administration of 5-HTP myoclonic response to finger taps was abolished in finger flexor muscles, but interpretation of the EMG response from biceps was difficult because of a large monosynaptic potential not previously present. The myoclonic response to median nerve stimulation in abductor pollicis brevis was abolished and the amplitudes of responses in finger flexors and biceps reduced by approximately $60 \%$.

\section{Discussion}

In this paper we have assumed that the spontaneous myoclonic jerks are equivalent to the elicited jerks and that their site of origin in the central nervous system is the same. The same muscles are involved in the same temporal sequence in both and the electromyographic activity in individual muscles and the electroencephalographic discharges are similar. It is reasonable to suppose that the spontaneous jerks are elicited by stimuli which have not been identified, but which are always present, although we cannot exclude the possibility of spontaneous discharges.

The jerks have been studied in averages or in series of single sweeps. As has been pointed out, averaged data can be confusing, for a component can become smaller because of an actual decline in size, less frequent appearance, increased jitter, or any combination. It was jitter of the muscle bursts that first drew our attention to the difficulty in interpreting averaged data. There was jitter of any particular muscle with respect to an eliciting stimulus, and there

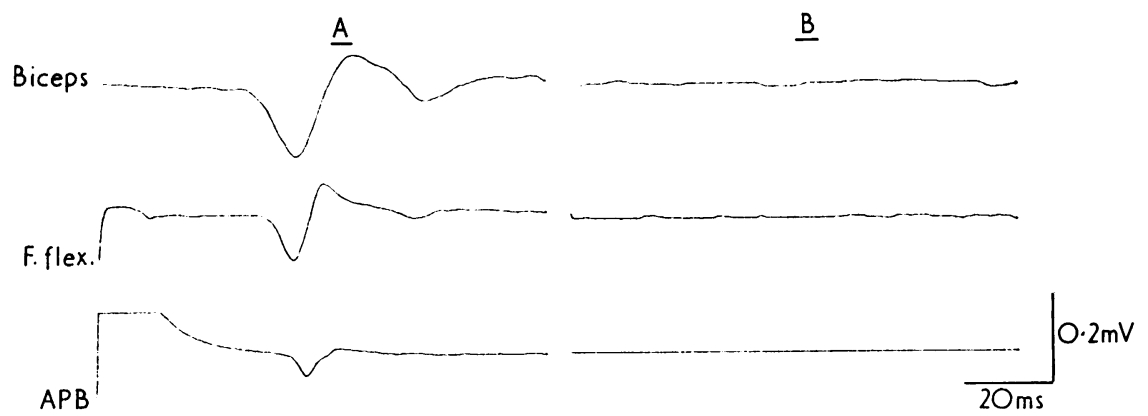

Fig. 8 A comparison of the response to mixed digital nerve stimulation. Electromyographic records, which are averages of 128 trials, are taken from biceps, finger flexors ( $F$. flex.) and abductor pollicis brevis $(A P B)$. In part $A$, the median nerve was stimulated at the wrist with $67 V$ for $100 \mu s$, which was below threshold for a direct motor response in $A P B$. In part $B$ the middle finger was stimulated with $90 \mathrm{~V}$ for $100 \mu$ s utilising ring electrodes. 


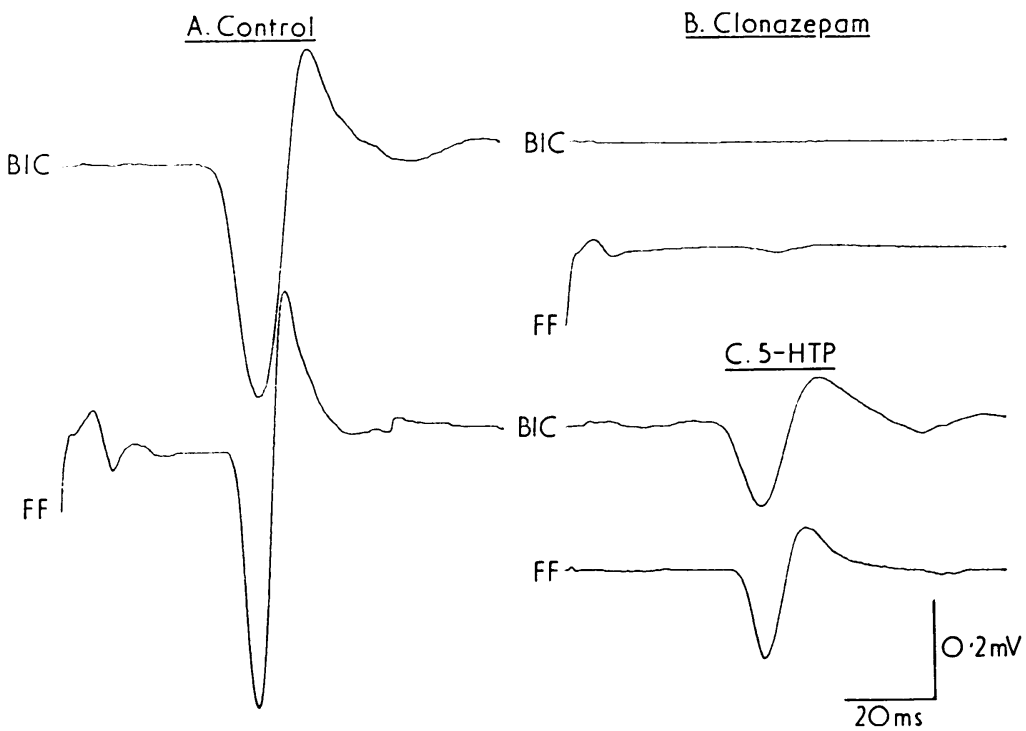

Fig. 9 Averaged myoclonic responses to median nerve stimulation at the wrist supramaximal for direct motor response in abductor pollicis brevis. Muscles recorded from are biceps $(B I C)$ and finger flexors $(F F)$. Averages are from 256 trials in parts $A$ and $C$, and 128 trials in part $B$. Part $A$ is the control response, part $B$ is the response after administration of clonazepam, and part $C$ is the response after administration of 5-HTP (see text for details).

was jitter of one muscle with respect to another. The further distal a muscle was from another, the greater was the jitter.

The phenomenon probably reflects the nature of the supraspinal organisation of the myoclonus-for example, variable spread in a complex polysynaptic network-since the signal sent to the spinal cord is powerful, producing absolutely stereotyped and almost synchronous firing of each muscle.

The times required for the neural signals to traverse peripheral motor nerves from point to point have already been noted (Table 1). The data from the myoclonic jerks gave values similar but slightly slower than those obtained with direct electrical stimulation. This and other data can also be used to make some deductions for approximate conduction times in central pathways mediating the myoclonus (Table 4).

The time from biceps to the cervical spinal cord can be estimated from the time for the monosynaptic tendon jerk $(15 \mathrm{~ms})$. Half of this time, about $8 \mathrm{~ms}$, was needed for the signal to go one way. The same time can be deduced and confirmed from other observations including the finger flexor monosynaptic stretch and the $F$ wave in abductor pollicis brevis, knowing the motor conduction delay from biceps to those muscles.

In similar fashion, the time from biceps femoris to the lumbar spinal cord can be estimated from the latency of the quadriceps tendon jerk, assuming quadriceps and biceps femoris are about equally distant from the cord (as seems to be the case; see, for example, Table 3). The tendon jerk latency was $25 \mathrm{ms,}$ which implies that the time from biceps femoris to the cord was about $13 \mathrm{~ms}$. Similar values can be calculated from the timing of the $\mathrm{H}$ reflex or the ankle jerk.

At least for the purpose of computing latencies, we can define a site in the central nervous system, $M$,

Table 4 Derived 'central conduction times'

\begin{tabular}{|c|c|c|}
\hline Site & Time $(m s)$ & Data used \\
\hline Cervical spinal cord to biceps & 8 & Biceps tendon jerk, finger flexor jerk, $F$ wave \\
\hline Lumbar spinal cord to biceps femoris & 13 & Quadriceps tendon jerk, ankle jerk, $\mathrm{H}$ reflex \\
\hline Cervical spinal cord to $M$ and back & 18 & $\begin{array}{l}\text { Latencies of myoclonic jerks in arm muscles after median nerve } \\
\text { stimulation and finger taps }\end{array}$ \\
\hline Cervical spinal cord to lumbar spinal cord & 2 & $\begin{array}{l}\text { Latencies of myoclonic jerks in leg muscles with respect to arm muscles } \\
\text { in spontaneous and median nerve elicited jerks }\end{array}$ \\
\hline Lumbar spinal cord to cervical spinal cord & 14 & $\begin{array}{l}\text { Comparison of latencies of myoclonic jerks in leg muscles after } \\
\text { median nerve stimulation and toe taps }\end{array}$ \\
\hline 11 th nucleus to 7 th nucleus & $\begin{array}{r}10 \\
9\end{array}$ & Latency in spontaneous jerks \\
\hline
\end{tabular}

M refers to the hypothetical source of the myoclonus in the central nervous system. 
where the myoclonus is 'generated'. As we know the time required for neural signals to travel back and forth from the spinal cord, the time from the cord to $M$ can be determined. The peripheral part of the efferent limb of the myoclonus consists of rapidly conducting fibres, just slightly slower than the most rapid motor fibres (Table 1). The peripheral part of the afferent limb also consists of rapidly conducting fibres as deduced from a comparison of median nerve stimulation at the wrist and elbow (see p. 259). The latency of the evoked myoclonic jerk in biceps after median nerve stimulation at the wrist was $39 \mathrm{~ms}$. Basing our calculations on motor conduction velocities, it would have taken about $6 \mathrm{~ms}$ for the afferent volley to reach the level of biceps, and, as already indicated, it would have taken $15 \mathrm{~ms}$ for the signal to traverse the segment biceps to cervical spinal cord and back. This leaves $18 \mathrm{~ms}$ for the loop from the spinal cord to $\mathbf{M}$ and back. A similar value can be calculated from the latency of the myoclonic jerk in biceps evoked by finger taps $(35 \mathrm{~ms})$. Subtracting $3 \mathrm{~ms}$ for the time taken for the afferent volley from the finger flexors to reach the level of biceps, and $15 \mathrm{~ms}$ for the segment biceps to spinal cord and back, leaves $17 \mathrm{~ms}$ for the spinal cord to M loop. Another method for calculating the time around this loop comes from a direct comparison of the latencies in abductor pollicis brevis of the $F$ wave $(32 \mathrm{~ms})$ and the myoclonic activity after median nerve stimulation at the wrist $(49 \mathrm{~ms})$. Since the $F$ wave latency reflects the time to the spinal cord and back, the excess latency of the myoclonic activity $(17 \mathrm{~ms})$ is the central loop time.

The time required for the efferent signal to traverse the spinal cord can be deduced from the difference in time of a myoclonic jerk appearing in the legs compared with one in the arms. In spontaneous jerks, biceps preceded biceps femoris by $5 \mathrm{~ms}$ and in jerks evoked by median nerve stimulation biceps preceded biceps femoris by $8 \mathrm{~ms}$ (average of these two values is about $7 \mathrm{~ms}$ ). Basing calculations on tendon jerk latencies (and other measures, $c f$. Table 4), it was $5 \mathrm{~ms}$ longer from the lumbar spinal cord to biceps femoris than from cervical spinal cord to biceps ( $13 \mathrm{~ms}-8 \mathrm{~ms})$. So it must take about $2 \mathrm{~ms}(7 \mathrm{~ms}-5 \mathrm{~ms})$ for the myoclonus signal to go from the cervical cord to the lumbar cord. Thus, the efferent conduction velocity for the myoclonic signal in the spinal cord is rapid, suggesting that the signal travels in a strongly influential, rapidly conducting oligosynaptic pathway.

The time that the afferent signal takes to traverse the spinal cord can be deduced from the difference in timing between jerks in the legs produced by stimulation of the arms and that produced by stimulation of the legs. The biceps femoris latency was $74 \mathrm{~ms}$ after toe taps and $44 \mathrm{~ms}$ after median nerve stimulation at the wrist (Table 3), a $30 \mathrm{~ms}$ difference. Some of this time is accounted for by a longer afferent path in the periphery. From values previously calculated, the time for median nerve at the wrist to cervical spinal cord was $14 \mathrm{~ms}$ and the time from toe flexors to lumbar cord was $30 \mathrm{~ms}$, a difference of $16 \mathrm{~ms}$. This leaves $14 \mathrm{~ms}$ as the afferent time from lumbar to cervical cord. This suggests that the afferent path in the spinal cord is much slower than the efferent path. If we assume that the long toe flexors and foot plantar flexors are also stimulated by toe taps, as they probably are, then the peripheral time in the leg is reduced and the apparent afferent cord conduction time further prolonged. This apparently long afferent signal time could be explained, at least in part, if muscle stretch takes longer to produce myoclonus than does mixed nerve stimulation; but this does not seem to be the case, as the latency of arm muscle response after finger taps was similar to the latency after median nerve stimulation (Table 3). Another explanation would be that it takes longer for afferent signals to be processed in $\mathbf{M}$ if they are from the legs than if they are from the arms.

Considering the cranial nerve musculature, the 11th nerve nucleus (trapezius and sternocleidomastoid) was activated first, followed by the 7th nerve nucleus (orbicularis oris) $10 \mathrm{~ms}$ later, and the 5th nerve nucleus (masseter) $19 \mathrm{~ms}$ later. Thus the signal producing the myoclonus seemed to travel (rather slowly) up the brain stem.

The appearance of the EMG burst in trapezius and sternocleidomastoid was the earliest electrophysiological event detectable in a myoclonic jerk. Taking half of the $8 \mathrm{~ms}$ required for the monosynaptic stretch reflex in sternocleidomastoid gives $4 \mathrm{~ms}$ for the time from the 11 th cranial nerve nucleus to its muscles. Recalling that biceps was $8 \mathrm{~ms}$ removed from the cervical cord (Table 4) and that the 11th nerve musculature preceded biceps by $8 \mathrm{~ms}$ (Table 2), we see that the 11 th nerve nucleus was activated $4 \mathrm{~ms}$ before the cervical spinal cord. In regard to cortical spikes, as the EMG burst in sternocleidomastoid preceded these by $1 \mathrm{~ms}$, the 11th nerve nucleus was activated $5 \mathrm{~ms}$ before the generation of the spike. Thus, activation of the 11 th nerve nucleus was the earliest phenomenon on the output side of the myoclonic jerk that we have identified. The myoclonus generating centre, $M$, must be closer to the 11 th nerve nucleus than to any other CNS structure examined.

The myoclonic jerks elicited in this case by mixed nerve electrical stimulation or tendon taps occurred after a delay too long for spinal cord mediated monosynaptic and other 'short latency' reflexes, yet too short for voluntary responses. They correspond to so-called 'long latency' reflexes. Some long latency reflexes can be mediated entirely in the spinal cord, while others, called long loop reflexes, require the 
participation of supraspinal structures. Several different long latency reflexes have been discovered in animals and man, and derangement of one (or more) of these may be responsible for the myoclonus.

The association of a type of myoclonus with such a reflex system would provide a rational system for classification of some of the different types of myoclonus.

The characteristics of the present case that seem important are:

1. The myoclonic jerk had a simple form and when it occurred it tended to involve all of the muscles in the body, including proximal muscles.

2. The EEG showed generalised spikes that were associated, but not time locked, to the jerk.

3. Afferent cord conduction time was long, while efferent cord conduction time was short.

4. Activation of cranial nerves was upward and the earliest event in the jerk could be localised in the medulla.

5. The critical stimulation for producing the myoclonus was muscle stretch.

6. The myoclonus was responsive to 5-HTP and clonazepam.

The first four characteristics point to a type of myoclonus mediated in the lower brain stem and probably in the reticular formation. Halliday (1975) has recently summarised the literature suggesting that the reticular formation may play an important role in myoclonus. The spinobulbospinal reflex, best studied in the cat, has tentatively been identified in man and is the only known long latency reflex mediated in the reticular formation (Shimamura et al., 1964; MeierEwert et al., 1972). The myoclonus in our patient, however, cannot be identified with that spinobulbospinal reflex, as the latter is characterised by fast afferent cord conduction and slow efferent cord conduction velocity. Rapid oligosynaptic pathways are known to originate in reticular formation and these must be involved in the kind of myoclonus under discussion here.

Other patients, with similar characteristics, have now been identified and we refer to this group as the 'reticular reflex myoclonus' type. They are contrasted with another group of patients who share the hypersynchronous EMG appearance of the myoclonus. In these patients the jerks are restricted to the area of stimulus, they have large sensory evoked potentials and the EEG correlate of the myoclonic jerk is a time-locked fragment of the sensory evoked potential. We refer to this group as the 'cortical loop reflex' type, as this seems to be the underlying mechanism. Details of the other patients will be reported elsewhere.

The fifth point in the above list characterising the present patient concerns the critical stimulus for the reflex myoclonus. As in the patient of Dawson (1947), stretch was the critical stimulus and simple touch was ineffective. On the other hand, for the patient of Sutton and Mayer (1974), touch was the critical stimulus and pure stretch was ineffective. We must conclude that both phenomena are possible, but then ask how this feature helps in the separation of different types of myoclonus. Theoretically, identification of the critical stimulus should help in identifying the reflex which has become deranged in producing the myoclonus. Practically, this does not seem useful yet, partly because the critical stimuli for the known long latency reflexes have not been well established. Additionally, even though both the present patient and Dawson's patient require stretch to produce the myoclonus, the present patient falls into the category of reticular reflex myoclonus while Dawson's patient would most probably be in the category of cortical loop reflex myoclonus. The patient of Sutton and Mayer would also most probably be in the cortical loop reflex category, yet the critical stimulus differs from that of Dawson's patient. These facts, together with the analysis of our other patients, suggest that critical stimulus is not a feature that separates the two identified categories. It may be, however, necessary eventually to subdivide these categories.

The excellent response of this patient's myoclonus to 5-HTP and clonazepam is shown by other patients with the physiological characteristics of the reticular reflex type of myoclonus (Chadwick et al., 1976). In such patients there is biochemical evidence that the myoclonus is related to a relative deficiency of brain serotonin (5-HT) and that the therapeutic effects of 5-HTP and clonazepam may be mediated by their action on 5-HT metabolism. In man the highest concentrations of 5-HT are found in the midbrain and medulla (Gottfries et al., 1974). Thus, the physiological and biochemical data are complementary in implicating brain-stem structures in the myoclonus of these patients. It is likely that the unknown reflex mediating the myoclonus is usually inhibited by the action of 5-HT systems of neurones.

We are grateful to the Medical Research Council, the National Fund for Research into Crippling Diseases, and the Bethlem Royal and Maudsley Hospitals Research Fund for financial support. We are also grateful to Mr H. C. Bertoya and Mr P. Asselman for expert assistance.

\section{References}

Chadwick, D., Hallett, M., Jenner, P., Reynolds, E. H., and Marsden, C. D. (1976). Factors predicting the 
response of myoclonus to drugs manipulating brain 5-hydroxytryptamine. Brain, in press.

Dawson, G. D. (1947). Investigations on a patient subject to myoclonic seizures after sensory stimulation. Journal of Neurology, Neurosurgery, and Psychiatry, 10, 141-162.

Gottfries, C. G., Roos, B. E., and Winblad, B. (1974). Determination of 5-hydroxytryptamine, 5-hydroxyindoleacetic acid and homovanillic acid in brain tissue from autopsy material. Acta Psychiatrica Scandinavica, 50, 496-507.

Halliday, A. M. (1967). The electrophysiological study of myoclonus in man. Brain, 90, 241-284.

Halliday, A. M. (1975). The neurophysiology of myoclonic jerking-a reappraisal. In Myoclonic Seizures (Excerpta Medica International Congress Series, No. 307), pp. 1-29. Edited by M. H. Charlton. Excerpta Medica: Amsterdam.
Marsden, C. D., and Parkes, J. D. (1973). Abnormal movement disorders. British Journal of Hospital Medicine, 10, 428-450.

Meier-Ewert, K., Hümme, U., and Dahm, J. (1972). New evidence favouring long loop reflexes in man. Archiv. für Psychiatrie und Nervenkrankheiten, 215, 121-128.

Shimamura, M., Mori, S., Matsushima, S., and Fujimori, B. (1964). On the spino-bulbo-spinal reflex in dogs, monkeys and man. Japanese Journal of Physiology, 14, 411-421.

Sutton, G. G. (1975). Receptors in focal reflex myoclonus. Journal of Neurology, Neurosurgery, and Psychiatry, 38, 505-507.

Sutton, G. G., and Mayer, R. F. (1974). Focal reflex myoclonus. Journal of Neurology, Neurosurgery, and Psychiatry, 37, 207-217. 